Reprinted F rom:

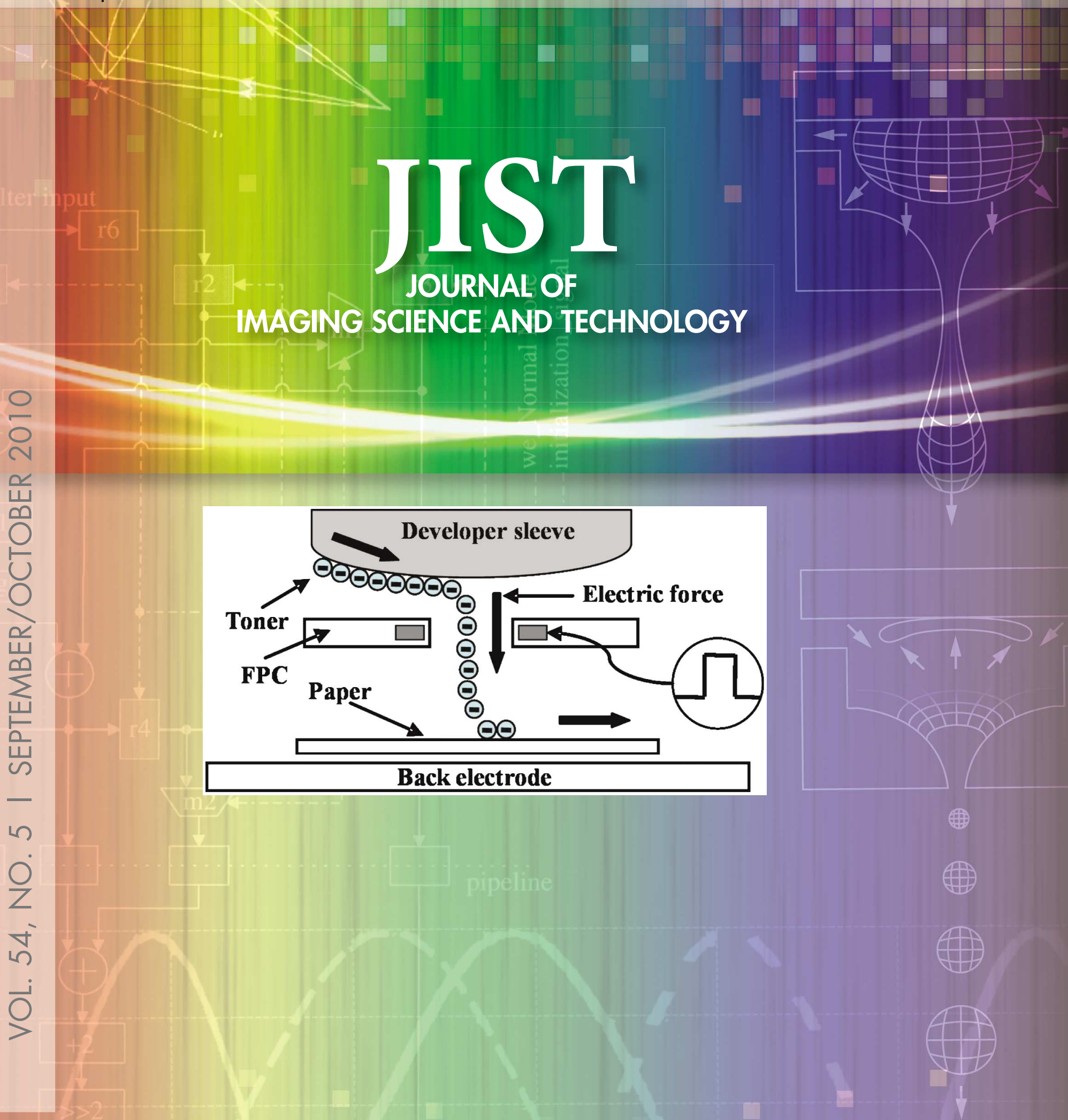




\title{
Predicting the Reflectance of Paper Dyed with Ink Mixtures by Describing Light Scattering as a Function of Ink Absorbance
}

\author{
Fabrice Rousselle, Mathieu Hébert and Roger D. Hersch ${ }^{\star}$ \\ Ecole Polytechnique Fédérale de Lausanne (EPFL), 1015 Lausanne, Switzerland \\ E-mail: rd.hersch@epfl.ch
}

\begin{abstract}
We consider the problem of predicting the spectral reflectance of paper samples immersed in ink mixtures of varying ink concentrations. Relying on an adapted version of the Kubelka-Munk theory, we predict the reflectances of the samples dyed by ink mixtures. We first derive a method to calculate the effective scattering coefficient of an inked paper sample as a function of its absorbance coefficient. Then we learn from a single sample the reduction in ink concentrations when two inks are mixed. Using these ink concentration reduction factors, we can then predict the reflectances of paper colored by ink mixtures of various nominal concentrations. (c) 2010 Society for Imaging Science and technology.

[DOI: 10.2352/J.ImagingSci.Technol.2010.54.5.050501]
\end{abstract}

\section{INTRODUCTION}

Ink jet printers use multiple inks which, thanks to halftoning, enable producing a wide color gamut. Pixel dot sizes are variable and can be chosen so as to produce lighter or stronger tones. Selecting the dot size for each ink and each pixel, combined with half-toning, yields a very rich set of color rendering options. In order to better assess this potential, one of the challenges is to predict the reflectance spectra for any combination of pixel dot size and half-tone dot surface coverage.

Spectral prediction of the reflectance of color half-tone prints is a difficult problem, mainly due to the interaction of ink and paper. When ink is deposited on a porous paper, part of it penetrates the paper and part of it remains on the surface. The ink concentration as a function of depth within the paper is unknown. Creating exact models of the reflectances of mixtures of multi-ink half-tones with variable pixel dot sizes is therefore difficult.

As a first step toward the goal of predicting the reflectance of ink mixtures within the paper, we consider a simplified setup where the ink concentration within the paper is assumed to be uniform. The paper is immersed for $30 \mathrm{~min}$ in a colored solution with a predetermined ratio of inks and then dried. By considering each ink separately at different concentrations, we establish a link between the ink absorbance and the ink concentration. This allows us to deduce

${ }^{\wedge}$ IS\&T member

Received Dec. 1, 2009; accepted for publication Jul. 3, 2010; published online Aug. 18, 2010.

$1062-3701 / 2010 / 54(5) / 050501 / 8 / \$ 20.00$. an effective concentration for a given nominal concentration. From the measured reflectance spectrum of a paper died with a mixture of two inks, we retrieve for each ink a constant concentration adaptation factor expressing the interaction between the two inks. We then predict the reflectances of ink mixtures at other ink concentrations. In this setup, the inks are uniformly distributed within the paper. Paper is considered as a strongly scattering, Lambertian medium. Therefore, the Kubelka-Munk (henceforth noted $\mathrm{KM}$ ) theory was chosen as the basis of our work.

\section{RELATED WORK}

One of the first approaches to model variable pixel dot sizes is the one proposed by Berns aiming at predicting the reflectance of a continuous tone dye diffusing thermal transfer printer. The print is formed by a nondiffusing ink layer behaving according to Beer's law, located on top of a diffusing substrate (paper). Variable dot sizes are modeled as variable ink concentrations and yield the corresponding proportional ink absorbances. ${ }^{1}$ Soler, Morovic, and Dumaux propose a spectral data driven interpolation and extrapolation model for deducing the reflectances of solid ink layer superpositions at various pigment loading, printed on top of PVC-like substrates. ${ }^{2}$ Van de Capelle and Meireson ${ }^{3}$ characterize ink half-tone layer superpositions by measuring the reflectances of individual ink half-tones printed on a given background at successively increasing surface coverages. The deduced wavelength dependent characterization parameters comprise "scattering" parameters, "absorption" parameters, and layer "interaction parameters." It is not known how the method could be extended to work efficiently with variable pixel dot size half-tone layers.

Arney and Alber ${ }^{4}$ developed a probability model relating optical properties of paper and inks to tone reproduction. Their model aimed at accounting for ink spreading and ink penetration into paper. Yang and Kruse ${ }^{5}$ studied the penetration of ink within paper. They considered that a part of the ink stays at the surface and another part penetrates the paper. For the ink penetration, they considered a constant concentration as well as a linear and an exponential concentration gradient, and studied their impact on the reflectance prediction. In a more recent work, Yang ${ }^{6}$ used the classical KM model with variable ink thicknesses accounting for vari- 
able pixel dot sizes for predicting the reflectances of ink jet prints on transparencies.

Recently, Rousselle et al. ${ }^{7}$ proposed a different approach, relying on an extended Clapper-Yule model, ${ }^{8,9}$ which modeled variable dot size by variable ink thicknesses. While these research approaches gave interesting insights into the effects of ink thickness variations and the interaction with the paper, they either did not provide sufficient accuracy or were not adapted to our problem, namely, the reflectance prediction of paper dyed with ink mixtures at variable concentrations.

The goal of the present work is to focus on the fundamental issue of predicting the reflectance spectra of the Neugebauer primaries obtained by mixtures of inks. Neugebauer primaries form the basic colorants used in most spectral prediction models. One may then develop approaches for predicting reflectances of multi-ink half-tones at variable ink pixel dot sizes.

\section{FUNDAMENTALS OF THE PROPOSED APPROACH}

We are interested in modeling the interaction of inks within a diffusing medium such as paper. We rely to the KM theory ${ }^{10}$ which is applicable to diffusing media. The main appeal of this theory is its simplicity. A sample is defined primarily by its wavelength dependent absorbing and scattering coefficients, respectively noted $K$ and $S$. Within the KM equations, they correspond to independent physical properties. However, it has been shown that these parameters are actually interdependent. ${ }^{11,12}$

Before considering the issue of the interdependence of the $K$ and $S$ coefficients and its impact on the prediction of reflectance spectra, let us review the actual equations ${ }^{10}$ allowing one to compute a sample reflectance $R$ and transmittance $T$ for each wavelength separately,

$$
\begin{gathered}
R=\frac{1-R_{g}(a-b \operatorname{coth} b S X)}{a+b \operatorname{coth} b S X-R_{g}}, \\
T=\frac{b}{a \sinh b S X+b \cosh b S X},
\end{gathered}
$$

where $X$ is the sample thickness, $R_{g}$ is the wavelength dependent reflectance of the backing, $a=(K+S) / S$, and $b=\sqrt{a^{2}-1}$. The thickness $X$ of an initial sample can be set arbitrarily to a value of unity. All other samples thicknesses will then be given relative to this initial sample thickness.

The parameters $K$ and $S$ are wavelength dependent and computing their values requires two spectral measurements. One may use the reflectance $R_{0}$ of a sample of finite thickness with no backing and the reflectance $R_{\infty}$ of a sample of infinite thickness. Then, the value of $a$ is obtained as follows: ${ }^{10}$

$$
a=\frac{\frac{1}{R_{\infty}}+R_{\infty}}{2} .
$$

Alternatively, we can compute the value of $a$ from the transmittance $T_{0}$ and the reflectance $R_{0}$ of a sample with no backing (equivalent to a completely black backing), ${ }^{10}$

$$
a=\frac{1+R_{0}^{2}-T_{0}^{2}}{2 R_{0}} .
$$

Applying $b=\sqrt{a^{2}-1}$, we compute $S$ and then $K$,

$$
\begin{gathered}
S=\frac{a \operatorname{coth} \frac{a-R_{0}}{b}-a \operatorname{coth} \frac{a}{b}}{b}, \\
K=(a-1) S .
\end{gathered}
$$

In this work, we will rely on the measured transmittance with no backing. The main reason behind this choice is that samples of infinite thickness are not always available. For instance, in the context of ink jet printing, no such sample could be produced. It is important to note that the accuracy of the computed values for $K$ and $S$ is highly dependent on the quality of the measurements. This is an issue because, in practice, the measured transmittance of samples is very low at wavelengths of high absorbance. Since the measurement noise magnitude is large with respect to the absolute measured value, this leads to numerical instability. Incidentally, use of the reflectance of an infinitely thick sample $R_{\infty}$ is also problematic since, for high absorbance, the reflectance of $R_{0}$ and $R_{\infty}$ will be very close and the measurement noise will have a magnitude similar to the difference between the two measurements, leading to widely varying results. In the worst case, due to the noise, the value of $R_{\infty}$ could be larger than the value of $R_{0}$. Solving the KM equations for $K$ and $S$ would then yield complex numbers. This imposes a serious limit on any method derived from the KM theory.

In this work, we are interested in ink mixtures. In many references, the absorbing and scattering powers of materials are assumed to be additive. ${ }^{13,14}$ According to that approach, the absorbance and scattering coefficients of an inked sample are obtained by summing the respective coefficients of the paper and the inks,

$$
\begin{aligned}
& K_{m}(\lambda)=K_{p}(\lambda)+\sum_{i=i n k s} \xi_{i} K_{i}(\lambda), \\
& S_{m}(\lambda)=S_{p}(\lambda)+\sum_{i=i n k s} \xi_{i} S_{i}(\lambda),
\end{aligned}
$$



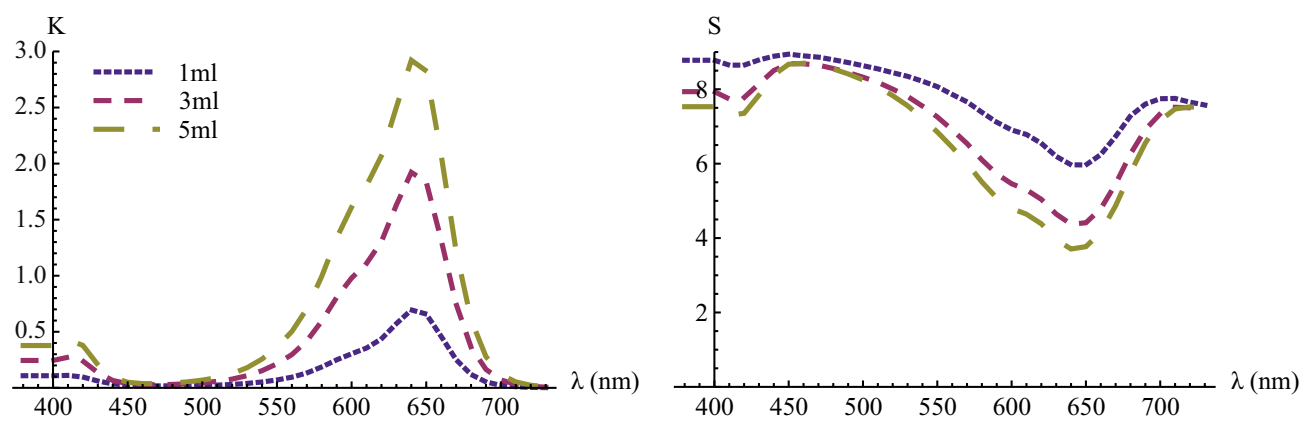

Figure 1. Absorbance and scattering coefficients of cyan paper samples at increasing ink concentrations ( 1 , 3 , and $5 \mathrm{ml}$ of ink in 39,37, and $35 \mathrm{ml}$ of water respectively). When the absorbance increases, the scattering coefficient decreases, underlying the link between the two parameters.
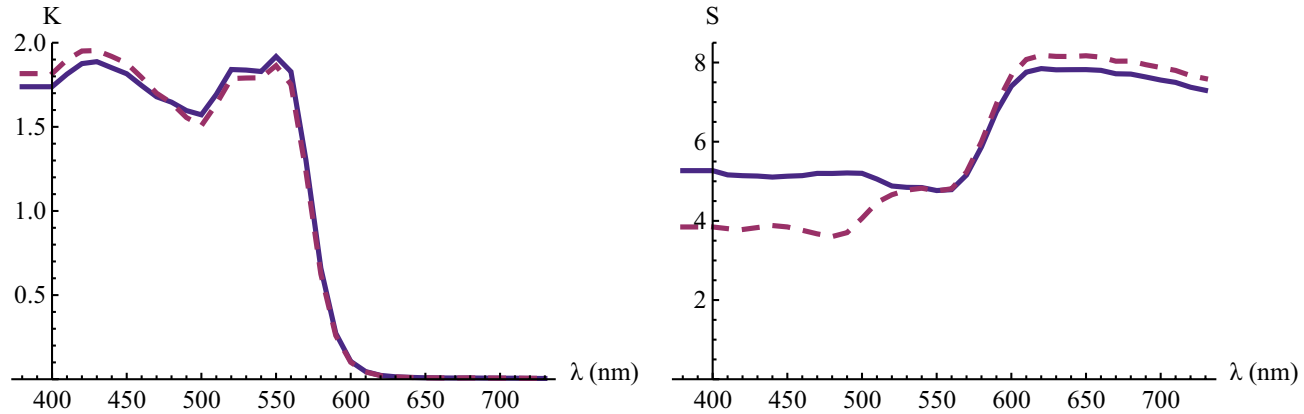

Figure 2. Absorbance and scattering coefficients of a red sample, predicted by summing the absorbance and scattering coefficients of the paper, the magenta ink and the yellow ink. The prediction is given by the dashed line and the measurement by the solid line. Note that the scattering approximation is rather poor in regions of high absorbance, while the approximation of the absorbance coefficient is satisfactory.

where $K_{p}$ and $S_{p}$ are, respectively, the absorbance and scattering coefficients of the paper, $K_{i}$ and $S_{i}$ the corresponding coefficients of the inks, and $\xi_{i}$ is the ink concentration. In practice however, these equations do not give a satisfactory approximation because of the interdependence between the $K$ and $S$ coefficients. To illustrate this issue, we take three samples prepared with different concentrations of a nondiffusing cyan ink. Since the ink is nondiffusing, it should only affect the absorbance coefficient, and not the scattering coefficient. In Figure 1, we see that in practice the two coefficients are affected. This interdependence implies that the simple formulas proposed in Eqs. (6) and (7) are not accurate. In Figure 2, we show that the scattering power of a red sample cannot be computed from the properties of the paper, the magenta and the yellow inks.

To address the interdependence of the $K$ and $S$ parameters, we follow the intuitive idea that increasing the absorbance of the inks reduces the scattering power of a sample. At one extremity of the absorbance range, the ink absorbance is null and the scattering coefficient is the scattering coefficient of the paper. At the other extremity, the ink absorbance is infinite and the scattering coefficient is null. In between, we assume that for significant absorbances, the effective scattering is the paper scattering scaled by a factor inversely proportional to the absorbance $K$, i.e., scaled in proportion to the absorption free mean path length $1 / K$ of light traversing a medium of absorbance $K$. The proposed formula is

$$
S=S_{p} \frac{S_{p}}{S_{p}+f(K)}
$$

where $S$ is the scattering coefficient of the colored sample (predicted or derived from measurement), $K$ is its absorbance coefficient and $S_{p}$ is the paper's intrinsic scattering coefficient and $f(K)$ is a smooth continuous function of $K$ accounting for small deviations from the assumed reduction in effective scattering. We assume that $\lim _{K \rightarrow 0} S=S_{p}$, i.e., a zero absorbance does not affect the scattering power of the paper. We also assume that $\lim _{K \rightarrow \infty} S=\mathbf{0}$, i.e., an infinite absorbance nullifies the paper's scattering power since all light entering the colored paper is absorbed. These two assumptions lead to the following boundary conditions: $f(K=0)=\mathbf{0}$ and $f(K=\infty)=\infty$. Between these two boundaries, the relation between $K$ and $S$, and therefore $f(K)$, is to be determined.

When the scattering coefficients of paper $S_{p}$ and of the colored sample $S$ are derived from measurements according to Eqs. (3) and (4), $f(K)$ can be calculated from Eq. (8) rearranged, 


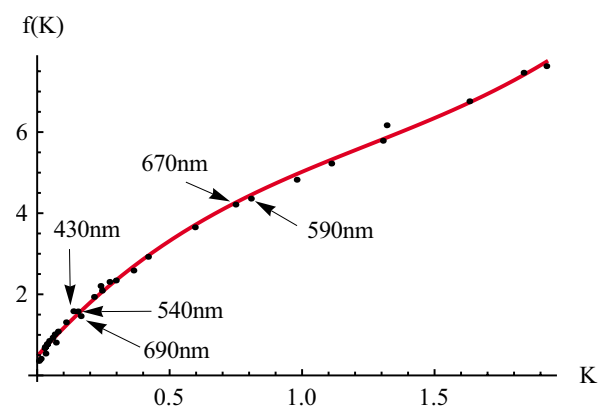

Figure 3. Relation between the $K$ values of a cyan colored sample and the corresponding $f(K)$ values. The cubic fit gives a good approximation.

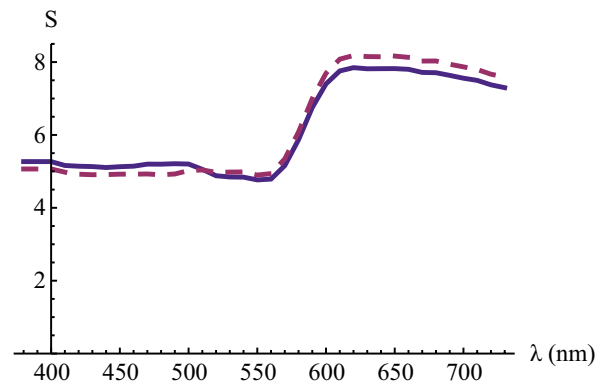

Figure 4. Measurement (solid line) of the scattering coefficient of a mixture of magenta and yellow inks and its prediction (dashed line) using $f(K)$. There is a clear improvement from the result of Fig. 2.

$$
f(K)=\frac{S_{p}^{2}-S \cdot S_{p}}{S} .
$$

We sort the values of $K$ and $f(K)$ according to $K$ independently of the wavelength to which they belong. For instance, if we have $K=1,3,2$ and $f(K)=a, b, c$, the ordered sets would be $1,2,3$ and $a, c, b$. Pairing these values $[K, f(K)]$ and plotting the resulting points, we can find an appropriate regression function. Figure 3 gives the resulting plot for a cyan ink and its approximation with a cubic function. In practice, we do not enforce $f(K=0)$ and obtain a better fit. In Fig. 3, the different points corresponding to different wavelengths are very close to the interpolation curve. This clearly shows that the relationship between $K$ and $f(K)$ and therefore between $S$ and $K$ does not depend on wavelength, but on the absorbance $K$ of the ink.
We call $f(K)$, the effective $K$ value. Assuming a separate mapping function $f_{i}(K)$ for each ink $i$, we model the effective $K$ coefficient of an ink mixture in paper by the sum,

$$
f\left(K_{m}\right)=\sum_{i=i n k s} f_{i}\left(\xi_{i m} K_{i}\right),
$$

where $\xi_{i m}$ is the concentration of ink $i$ within the mixture $m$. Using this approximated value of $f\left(K_{m}\right)$, we can then predict the scattering coefficient $S_{m}$ of the mixture according to Eq. (8). The absorbance coefficient of the mixture is computed according to Eq. (6). Now that we have the absorbance and scattering coefficients of the ink mixture in paper, the reflectance of the sample may be computed from Eq. (1) with $R_{g}=\mathbf{0}$.

In the same case as in Fig. 2, assuming $\xi_{i m}=\mathbf{1}$ for both inks, we obtain the result of Figure 4 . We see that the predicted scattering coefficient has been strongly improved. Using this new approach to predict the mixture reflectance yields a CIELAB error of $\Delta E_{94}=1.36$, compared to an error of $\Delta E_{94}=3.11$ previously. We further improve the quality of our approximation with a single fit of scaling factors giving the effective concentrations of the inks in the mixture at given nominal concentrations. This accounts for the interaction of inks within the mixture. Assuming this interaction is similar at other concentrations, we use it to predict the reflectances of the new mixture samples. For instance, learning from a red mixture at nominal concentrations $\xi_{m}=\mathbf{1}$ and $\xi_{y}=1$ of magenta and yellow inks, we predict the mixture reflectance for new arbitrary values of $\xi_{m}$ and $\xi_{y}$.

The KM theory does not take into account the interfaces of the sample with the air. These interfaces, as reported by Saunderson, ${ }^{15}$ induce a surface reflection as well as internal reflections between the colored paper and the air according to Fresnel's laws. We account for these interfaces, by modeling them using Kubelka's ${ }^{16}$ layering model. ${ }^{17}$ Using this model, we bound the paper with two paper-air interfaces. The characteristics of these two interfaces (surface reflection, internal reflection and transmittance) are identical and given by Snell's and Fresnel's formulae. As a first step of our computations, we calculate the intrinsic reflectances and transmittances of colored paper samples by removing the contribution of these interfaces. Details are given in Appendix A.
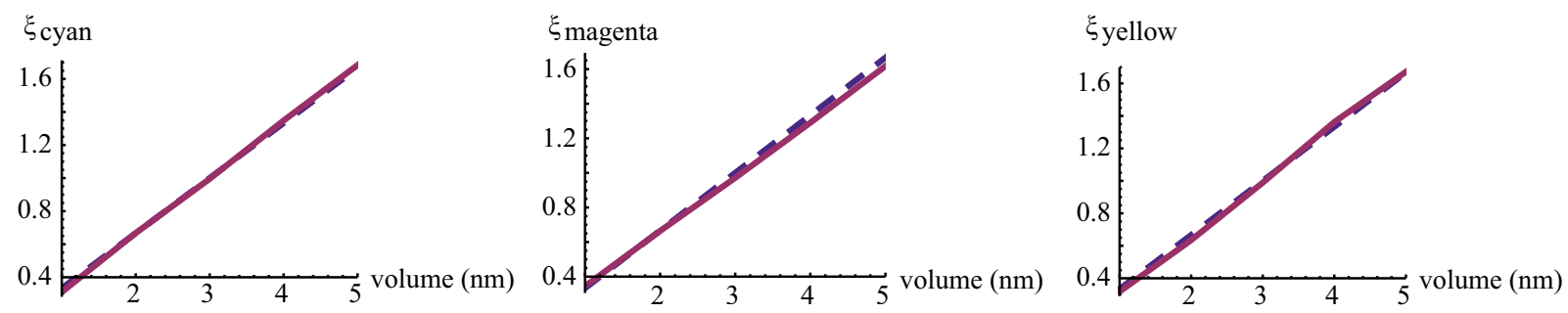

Figure 5. Effective cyan, magenta and yellow ink concentrations fitted from sample measurements. The $3 \mathrm{ml}$ ink volume was used as the reference measurement. The dashed line is the ideal linear scale and the solid line is the fitted concentration. 


\section{BACKWARD AND FORWARD PREDICTIONS}

In backward predictions, given a reflectance measurement, the goal is to deduce the ink concentrations in a mixture. The absorbances $K_{i}$ of the individual inks are computed from the KM calibration measurements at an initial relative ink concentration of 1 . It becomes possible to fit concentrations of inks by minimizing the root mean square difference between the measured and predicted spectra components.

In forward predictions, the goal is to predict the reflectance of a sample given its nominal ink concentrations. We use the term nominal to account for the concentration deviations when producing samples of varying ink concentration. We start by measuring a set of five samples for each individual ink covering the range of concentrations to be used in the mixtures. For each nominal concentration, we perform a backward prediction yielding the corresponding effective concentration. Figure 5 gives the effective concentration obtained for the cyan, magenta, and yellow inks. Once we have these nominal to effective concentration relations, we prepare a sample using a reference mixture where the ink concentrations are at the midpoint of the range. On this sample, we apply our backward prediction model to compute the effective concentrations of the inks in this mixture. This yields a scaling factor in respect to the single ink effective concentration. We obtain for the effective concentration of ink $i$ in the mixture,

$$
\xi_{i m}\left(N_{i}\right)=\xi_{i}\left(N_{i}\right) \cdot q_{i m},
$$

where $N_{i}$ is the nominal concentration, $\xi_{i}\left(N_{i}\right)$ is the effective concentration of the single ink $i$ at nominal concentration $N_{i}$, and $q_{i m}$ is the scaling factor of ink $i$ in mixture $m$. We can now compute $K_{m}$ according to Eq. (6), $S_{m}$ according to Eqs. (8) and (10), and the actual mixture reflectance $R_{m}$ according to Eq. (1).

\section{EXPERIMENTAL SETUP}

As mentioned in the Introduction, we work with a simplified setup where the ink penetrates completely and uniformly into the paper. We use a set of Colorex watercolor inks from Pebeo: cyan, magenta, and primary yellow. We extended this set by preparing light cyan and light yellow inks. This was done by diluting the original cyan and yellow inks in an equal volume of water, effectively halving their concentration. All spectral measurements were performed with the Color i7 spectrophotometer from GretagMacbeth, in reflectance mode according to the $\left(d: 8^{\circ}\right)$ geometry and in transmittance mode according to the $\left(d: 0^{\circ}\right)$ geometry.

For each ink, we first prepare a set of five samples covering the considered range of concentrations. These five samples are prepared by diluting $1,2,3,4$, and $5 \mathrm{ml}$ of ink with water to produce the $40 \mathrm{ml}$ solutions used for coloring the paper. From the measurements of the resulting five colored paper samples, we obtain the effective ink concentrations $\xi_{i}$ for each nominal concentration. We then prepare a set of five colored paper samples with mixtures of increasing uniform ink concentrations. For instance, a red mixture is prepared with the magenta and yellow inks. To produce the full set, we dilute $1 \mathrm{ml}$ of each of the two inks in $38 \mathrm{ml}$ of water, then $2 \mathrm{ml}$ of each of the two inks in $36 \mathrm{ml}$ of water, etc. From the midrange sample, using $3 \mathrm{ml}$ of each ink, we compute the mixture scaling factors $q_{i m}$.

We obtain colored samples by soaking a $25 \mathrm{~cm}^{2}$ square sheet of Biotop paper (paper without fluorescent brightening agents) in each solution. This is done by first depositing the sheet on the solution and letting it float for $15 \mathrm{~min}$. The sample is gradually filled with the solution. We then flip the sample and immerse it in the solution for another $15 \mathrm{~min}$. The sample is then dried by hanging it vertically. All droplets forming at the tip of the sample are removed at the beginning of the drying phase. Removing the extra droplets is crucial in order to create a homogeneous distribution of ink.

Once dried, each sample reflectance and transmittance is measured on both sides. Since the samples are supposed to be homogeneous, the average from the measurements of both sides is used to represent a single sample. In practice, the reflectance measurements of the two sides are indeed very close: we have an average CIELAB $\Delta E_{94}$ distance of 0.26 for cyan, 0.63 for magenta, and 0.11 for yellow.

We prepared three sets of mixtures: purple (light cyan and magenta), red (magenta and yellow), and light green (light cyan and light yellow). For the purple samples, the CIELAB distances between measured and predicted spectra are, from the lowest to the highest ink concentrations $\Delta E_{94}=(0.22,0.92,1.4,1.5,0.81)$. For the red samples, the distances are $\Delta E_{94}=(0.88,0.47,0.27,1.0,0.49)$. For the light green samples, the distances are $\Delta E_{94}=(0.33,0.29,0.20,0.32,0.16)$. Figure 6 shows the predicted and measured spectra for these three mixtures. $\mathrm{Nu}$ merical values used to produce these figures are given in the Appendixes B and C, Tables II-IV.

\section{CONCLUSION}

We propose a new approach for predicting the reflectance of a paper sample colored with a mixture of inks. We are able to demonstrate the validity of the approach on mixtures of two inks of varying concentrations by predicting their reflectance spectra with a high accuracy. The main limitation of our approach is that it badly handles high absorbances. In such cases, at some wavelengths, the transmittance measurement during KM model calibration is close to zero and comparable with the measurement noise. This limitation is inherent to the KM theory which does not allow computing accurately such extreme cases.

The main contribution of our work is, within the context of the KM theory, the prediction of the scattering power of a mixture of inks within a diffusing medium (paper). The direct approach of summing the scattering coefficients of the inks and the medium does not give a satisfactory approximation in regions of high absorbance. Instead, we rely on a separate measurement of the paper's intrinsic scattering coefficient which we combine with the inked paper sample absorbance coefficient to predict the colored sample scattering coefficient. In order to accurately predict the reflectances of paper colored with mixtures of inks of various concentra- 
Rousselle, Hebert, and Hersch: Predicting the reflectance of paper dyed with ink...
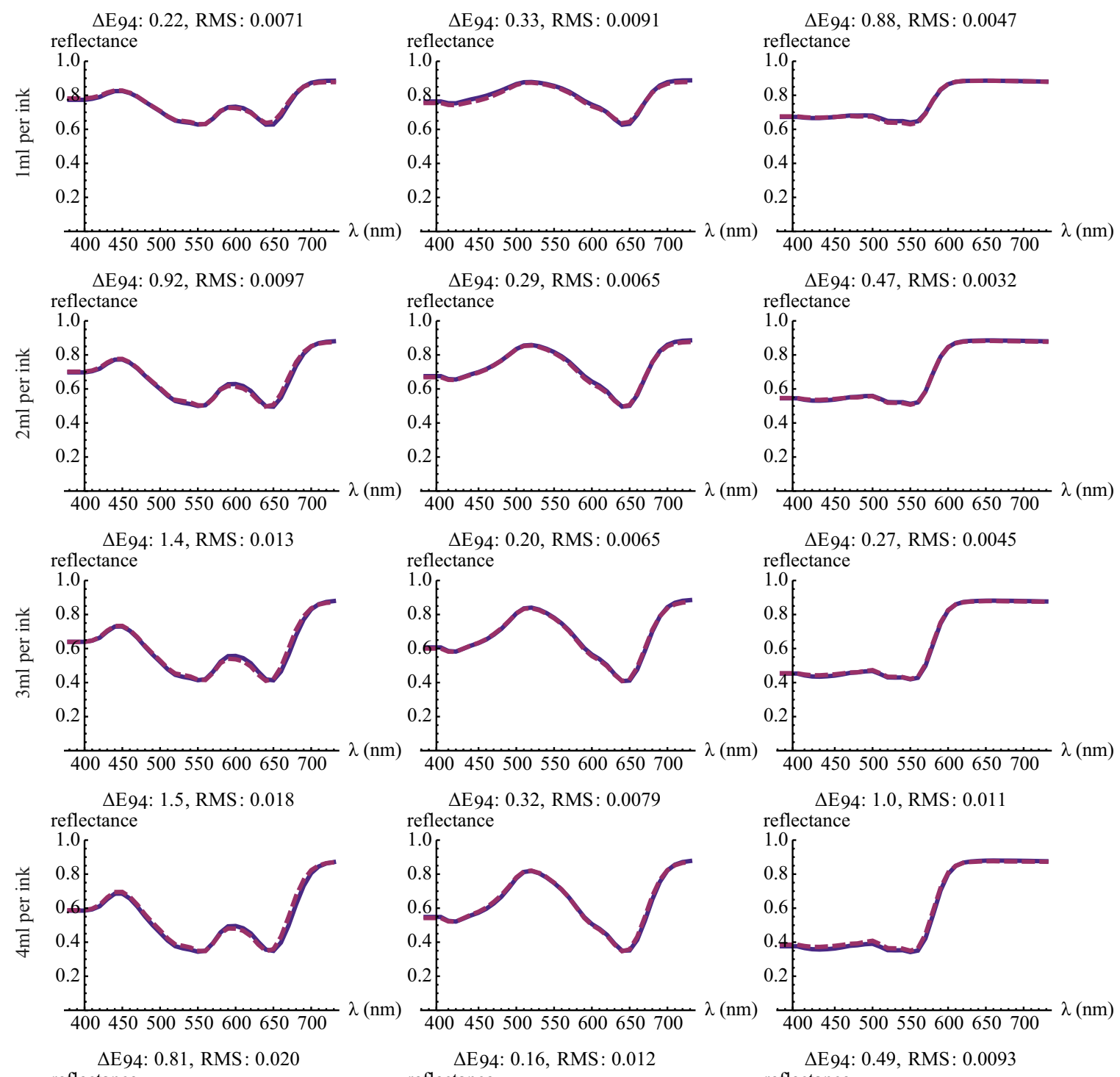

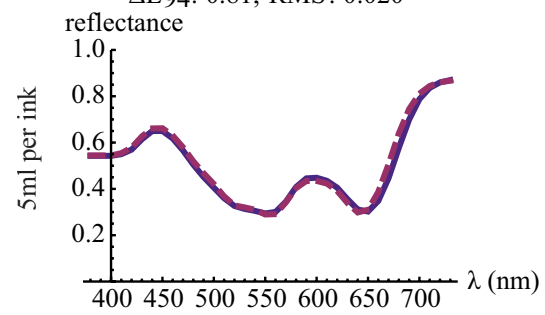

(a)

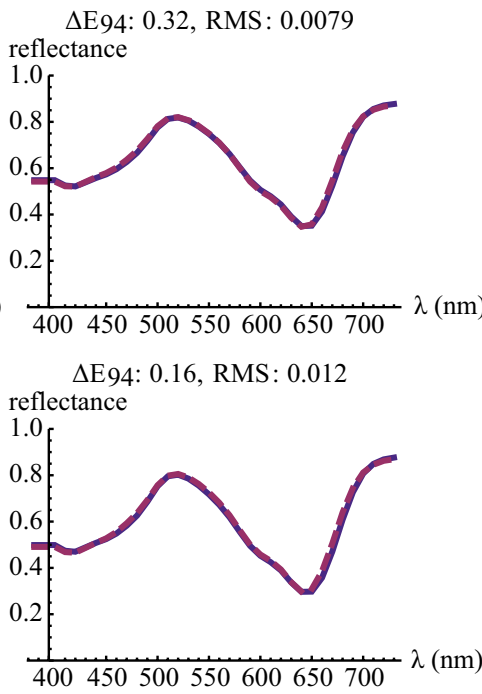

(b)

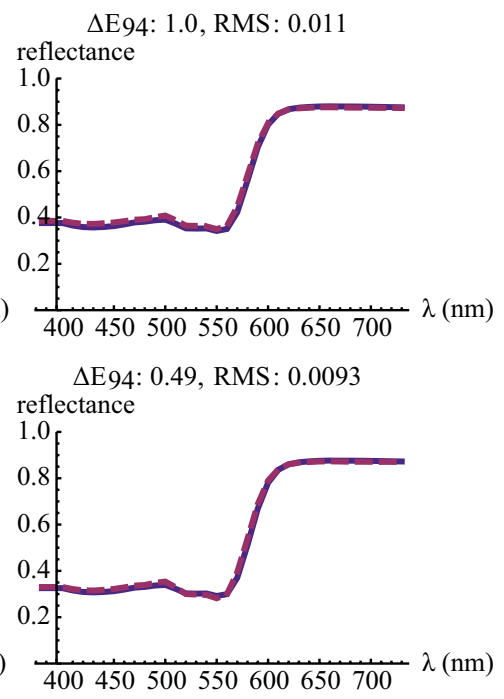

(c)

Figure 6. Prediction accuracy for (a) purple, (b) light green, and (c) red colored paper sets. The measurement is traced with a solid blue line and the prediction is traced with a dashed red line. The root mean square (RMS) distance is the metric used in the optimization process when calibrating our model.

tions, we introduce for each ink of the mixture a constant concentration scaling factor, fitted to a single ink mixture sample. As future work, we would like to extend the present approach to mixtures of more than two inks and to apply it to ink jet prints where ink is located both at the surface and inside the paper bulk.

\section{ACKNOWLEDGMENTS}

We would like to thank the Swiss National Science Foundation for their support, Grant No. 200020-126757/1.

\section{APPENDIX A: ACCOUNTING FOR AIR INTERFACES}

We consider that a sample is bounded by two symmetric paper-air interfaces. In the Kubelka layering model, ${ }^{16}$ a layer is defined by its reflectance and transmittance, which differ depending on top to bottom and bottom to top light fluxes. We note $R_{1}$ the top to bottom reflectance of a layer and $R_{I}$ its bottom to top reflectance. Similarly, we define the top to bottom transmittance $T_{1}$ and the bottom to top transmittance $T_{I}$. We have similar notations for the second and third 
Table I. Effective ink concentrations for nominal ink volumes of 1, 2, 3, 4, and $5 \mathrm{ml}$ in a total volume of ink + water of $40 \mathrm{ml}$. The $3 \mathrm{ml}$ ink volume was used for each ink as the reference measurement for the $K$ coefficient.

\begin{tabular}{llllll}
\hline Ink volume & $1 \mathrm{ml}$ & $2 \mathrm{ml}$ & $3 \mathrm{ml}$ & $4 \mathrm{ml}$ & $5 \mathrm{ml}$ \\
\hline Cyan & 0.31 & 0.67 & 1.00 & 1.35 & 1.68 \\
Magenta & 0.34 & 0.66 & 0.97 & 1.29 & 1.62 \\
Yellow & 0.32 & 0.64 & 0.99 & 1.36 & 1.67 \\
Light cyan & 0.34 & 0.65 & 1.01 & 1.36 & 1.60 \\
Light yellow & 0.37 & 0.66 & 1.00 & 1.40 & 1.78 \\
\hline
\end{tabular}

Table II. Purple mixture. Effective ink concentrations $\xi_{\text {im }}$ for nominal ink volumes of l- $5 \mathrm{ml}$, mixture scaling factors $q_{i m}$ and absorbance mapping functions $f_{i}(K)$. Indices $P, C$, and $M$, respectively, correspond to purple, light cyan, and magenta.

\begin{tabular}{lc}
$\xi_{c P}$ & $0.31,0.64,0.96,1.26,1.54$ \\
$q_{c P}$ & 0.95 \\
$f_{c}(K)$ & $0.431+6.36 x-2.07 x^{2}+0.376 x^{3}$ \\
$\xi_{M P}$ & $0.31,0.59,0.87,1.16,1.45$ \\
$q_{M P}$ & 0.89 \\
$f_{M}(K)$ & $0.0512+7.37 x-3.10 x^{2}+0.711 x^{3}$ \\
\hline
\end{tabular}

Table III. Red mixture. Effective ink concentrations $\xi_{\text {im }}$ for nominal ink volumes of $1-5 \mathrm{ml}$, mixture scaling factors $q_{i m}$ and absorbance mapping functions $f_{i}(K)$. Indices $R, M$, and $Y$, respectively, correspond to red, magenta, and yellow.

\begin{tabular}{lc}
$\xi_{M R}$ & $0.35,0.67,0.99,1.31,1.65$ \\
$q_{M R}$ & 1.01 \\
$f_{M}(K)$ & $0.0512+7.37 x-3.10 x^{2}+0.711 x^{3}$ \\
$\xi_{Y R}$ & $0.27,0.56,0.85,1.15,1.46$ \\
$q_{Y R}$ & 0.87 \\
$f_{Y}(K)$ & $0.316+6.46 x-1.97 x^{2}+0.398 x^{3}$ \\
\hline
\end{tabular}

Table IV. Light green mixture. Effective ink concentrations $\xi_{\text {im }}$ for nominal ink volumes of $1-5 \mathrm{ml}$, mixture scaling factors $q_{i m}$ and absorbance mapping functions $f_{i}(K)$. Indices $g, c$, and $y$, respectively, correspond to light green, light cyan, and light yellow.

\begin{tabular}{lc}
$\xi_{c g}$ & $0.31,0.65,0.97,1.271 .55$ \\
$q_{c g}$ & 0.96 \\
$f_{c}(K)$ & $0.431+6.36 x-2.07 x^{2}+0.376 x^{3}$ \\
$\xi_{y g}$ & $0.36,0.67,1.01,1.39,1.80$ \\
$q_{y g}$ & 1.01 \\
$f_{y}(K)$ & $0.0348+7.94 x-6.28 x^{2}+2.63 x^{3}$ \\
\hline
\end{tabular}

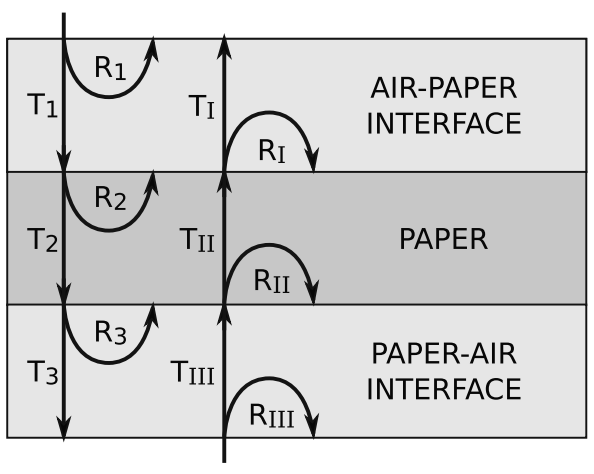

Figure 7. The two symmetric paper-air interfaces bounding the paper layer with their respective reflectances and transmittances.

layers. In our case, the first and third layer are the symmetric paper-air interfaces. Figure 7 illustrates the setup.

The top to bottom reflectance and transmittance of two layers is calculated as follows: ${ }^{16}$

$$
\begin{gathered}
T_{1,2}=\frac{T_{1} T_{2}}{1-R_{I} R_{2}}, \\
R_{1,2}=R_{1}+\frac{T_{1}^{2} R_{2}}{1-R_{I} R_{2}} .
\end{gathered}
$$

By simply inverting layers 1 and 2 and setting the appropriate bottom to top reflectances, we obtain the bottom to top reflectance and transmittance. Applying this iteratively, we obtain the cumulative reflectances and transmittances of the three layers. We have the following characteristics for the first and third layer:

$$
\begin{gathered}
R_{1}=R_{I I I}=R_{s} ; R_{I}=R_{2}=R_{i}, \\
T_{1}=T_{I I I}=T_{\text {in }} ; T_{I}=T_{2}=T_{e x},
\end{gathered}
$$

where $R_{s}$ is the specular reflection at the air-paper interface, $R_{i}$ is the internal reflection at the paper-air interface, $T_{i n}$ is the transmittance of the interface for the incoming light, and $T_{e x}$ is the transmittance of the interface for the outgoing light. Applying Fresnel's formula, we obtain for a diffuse light source: ${ }^{18,19} R_{s}=0.04, R_{i}=0.6, T_{i n}=0.9$, and $T_{e x}=0.4267$, with a diffraction index of air $n_{\text {air }}=1$ and as- 


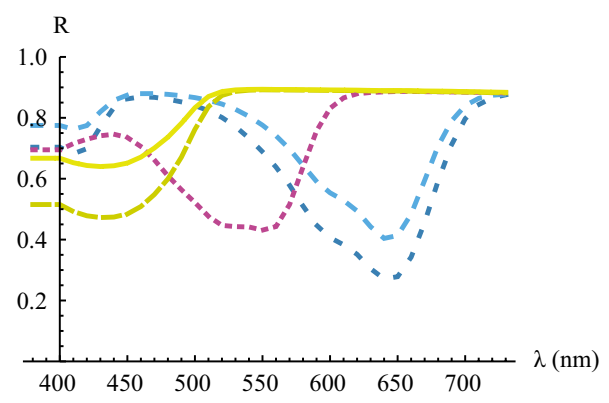

Figure 8. Reflectances of the cyan and light cyan (short dashes), magenta (dotted line), yellow (continuous), and light yellow samples (long dashes). All measurements were done on the sample produced with a nominal ink volume of $3 \mathrm{ml}$.

suming that the diffraction index of the inked paper is $n_{\text {paper }}=1.5$.

We measure the cumulative top to bottom and bottom to top reflectances and transmittances with the spectrophotometer. We solve a linear equation system and obtain the following formulas:

$$
\begin{aligned}
& d=\left(R_{i}\left(R_{s}-R_{T B}\right)-T_{e x} T_{i n}\right)\left(R_{B T} R_{i}-R_{i} R_{s}+T_{e x} T_{i n}\right) \\
&+R_{i}^{2} T_{B T} T_{T B}, \\
& R_{2}=\frac{\left(R_{s}-R_{T B}\right)\left(R_{B T} R_{i}-R_{i} R_{s}+T_{e x} T_{i n}\right)+R_{i} T_{T B} T_{B T}}{d}, \\
& R_{I I}=\frac{\left(R_{B T}-R_{s}\right)\left(R_{i}\left(R_{s}-R_{T B}\right)-T_{e x} T_{i n}\right)+R_{i} T_{B T} T_{T B}}{d}, \\
& T_{2}=\frac{-\left(T_{B T} T_{e x} T_{i n}\right)}{d}, \\
& T_{I I}=\frac{-\left(T_{e x} T_{i n} T_{T B}\right)}{d},
\end{aligned}
$$

where $R_{T B}$ and $T_{T B}$ are the top to bottom cumulative reflectance and transmittance of the three layers and $R_{B T}$ and $T_{B T}$ are the bottom to top cumulative reflectance and transmittance of the three layers. We assume the ink is homogeneously distributed in the sample and use the average of the measurements from the two sides $R_{T B}^{\prime}=R_{B T}^{\prime}=R_{a v g}$ and $T_{T B}^{\prime}=T_{B T}^{\prime}=T_{\text {avg, }} \quad$ with $\quad R_{\text {avg }}=\left(R_{T B}+R_{B T}\right) / 2 \quad$ and $T_{\text {avg }}=\left(T_{T B}+T_{B T}\right) / 2$.

The internal reflectance of the colored paper is $R_{2}$, and its internal transmittance is $T_{2}$. These two values are used in Eqs. (1) and (2).

\section{APPENDIX B: NUMERICAL VALUES FOR THE EXPERIMENT}

Five inks were used to prepare the test mixtures: cyan, magenta, yellow, light cyan, and light yellow. Fig. 5 shows the nominal to effective concentration curves for the cyan, magenta, and yellow inks. Table I lists the exact values for the relationships between nominal volume and effective concentrations of single inks. Figure 8 gives the reflectances of the cyan, light cyan, magenta, yellow, and light yellow inks at the $3 \mathrm{ml}$ nominal ink volume.

\section{APPENDIX C: NUMERICAL RESULTS FOR TEST MIXTURES}

Tables II-IV list the effective ink concentrations within the three test mixtures, the concentration scaling factors, as well as the absorbance coefficient mapping functions.

\section{REFERENCES}

${ }^{1}$ R. S. Berns, "Spectral modeling of a dye diffusion thermal transfer printer (Journal Paper)", J. Electron. Imaging 2, 359-370 (1993).

${ }^{2}$ P. Soler, J. Morovic, and H. Doumaux, "Spectral Data-Driven Model for Overprint Estimation”, Proc. IS\&T/SID’s 16th Color Imaging Conference (IS\&T, Springfield, VA, 2008) pp. 89-92.

${ }^{3}$ J. P. Van de Capelle and B. Meireson, in Colour Imaging: Vision and Technology, edited by L. W. MacDonald and M. R. Luo (Wiley, New York, 1999), pp. 179-188.

${ }^{4}$ J. S. Arney and M. L. Alber, "Optical effects of ink spread and penetration on halftones printed by thermal ink jet", J. Imaging Sci. Technol. 42, 331-334 (1998).

${ }^{5} \mathrm{~L}$. Yang and B. Kruse, "Ink penetration and its effects on printing", Proc. SPIE 3963, 365-375 (2000).

${ }^{6} \mathrm{~L}$. Yang, "Characterization of inks and ink application for ink-jet printing: model and simulation", J. Opt. Soc. Am. A 20, 1149-1154 (2003).

${ }^{7}$ F. Rousselle, T. Bugnon, and R. D. Hersch, "Spectral prediction model for variable dot-size printers", Proc. IS\&T/SID's 16th Color Imaging Conference (IS\&T, Springfield, VA, 2008) pp. 73-78.

${ }^{8}$ F. R. Clapper and J. A. C. Yule, "The effect of multiple internal reflections on the densities of half-tone prints on paper", J. Opt. Soc. Am. 43, 600-603 (1953).

${ }^{9}$ R. D. Hersch, P. Emmel, F. Collaud, and F. Crété, "Spectral reflection and dot surface prediction models for color halftone prints", J. Electron. Imaging 14, 033001-1-033001-12 (2005).

${ }^{10} \mathrm{P}$. Kubelka, "New contributions to the optics of intensely light-scattering materials. Part I", J. Opt. Soc. Am. 38, 448-448 (1948).

${ }^{11}$ J. H. Nobbs, "Kubelka-Munk theory and the prediction of reflectance", Rev. Prog. Color. Relat. Top. 15, 66-75 (1985).

${ }^{12}$ L. Yang and B. Kruse, "Revised Kubelka-Munk theory. I. Theory and application”, J. Opt. Soc. Am. A 21, 1933-1941 (2004).

${ }^{13}$ J. H. Nobbs, "Colour-match prediction for pigmented materials", in Colour Physics for Industry, edited by R. McDonald (Society of Dyers and Colorists, Bradford, U.K., 1997), pp. 292-371.

${ }^{14} \mathrm{E}$. Allen, "Basic equations in computer color matching, II. Tristimulus match, two-constant theory", J. Opt. Soc. Am. 64, 991-993 (1974).

${ }^{15} \mathrm{~J}$. L. Saunderson, "Calculation of the color of pigmented plastics", J. Opt. Soc. Am. 32, 727-729 (1942).

${ }^{16} \mathrm{P}$. Kubelka, "New contributions to the optics of intensely light-scattering materials. Part II: Nonhomogeneous layers", J. Opt. Soc. Am. A 44, 330-335 (1954).

${ }^{17}$ M. Hébert and R. D. Hersch, "Reflectance and transmittance model for recto-verso halftone prints", J. Opt. Soc. Am. A 23, 2415-2432 (2006).

${ }^{18}$ D. B. Judd, "Fresnel Reflection of Diffusely Incident Light", J. Res. Natl. Bur. Stand. 29, 329-332 (1942).

${ }^{19}$ P. Emmel, in Digital Color Imaging Handbook, edited by G. Sharma (CRC, Boca Raton, FL, 2003), p. 180. 
(C)2010 Society for Imaging Science and Technology (IS\&T)

All rights reserved. This paper, or parts thereof, may not be reproduced in any form without the written permission of IS\&T, the sole copyright owner of The Journal of Imaging Science and Technology.

For information on reprints or reproduction contact

Donna Smith

Production Editor

The Journal of Imaging Science and Technology

Society for Imaging Science and Technology

7003 Kilworth Lane

Springfield, Virginia 22151 USA

703/642-9090 extension 107

703/642-9094 (fax)

dsmith@imaging.org

www.imaging.org 